\title{
A Novel Contactless Rotary Position Micro-Sensing System Based on Electromagnetism
}

\author{
Lv Bohan \\ The 18th Institute China Academy of Launch Vehicle \\ Technology \\ Beijing, China \\ Lbh19881230@126.com \\ Liu Shan \\ The 18th Institute China Academy of Launch Vehicle \\ Technology \\ Beijing, China
}

\author{
Zeng Guangshang \\ The 18th Institute China Academy of Launch Vehicle \\ Technology \\ Beijing, China
}

Qu Xiyan

Harbin University of Science and Technology

Harbin, China

\begin{abstract}
Based on the law of electromagnetic induction, a novel contactless absolute angular position micro-sensor has been designed in the literature. It is mainly composed of three parts: a magnet having two portions shaped as circle segments with different center points, a Hall effect sensor and a signal processing circuit. The linearity of the sensing system is improved by particularly designing spatial magnetic field distribution of asymmetric magnet, and Hall output signal is converted by the second-order low-pass filter circuit into linear analog voltage. Experiment has been performed on a prototype of the angular position sensor and the results demonstrate the proposed scheme is feasible. Theoretic precision and testing error analysis of the sensor has been pursued at the end of the paper. The proposed sensor has linear characteristics with accuracy below $\pm 2.5^{\circ}$ and linearity with $R^{2}$ of 0.9992 , within the measurement range of 0 to $150^{\circ}$.
\end{abstract}

Keywords-Absolute angular position sensor; Robot control; Electromagnetic sensor; micro-sensor

\section{INTRODUCTION}

The development of robot and automation techniques has been attracting great attentions of many investigators for ages, which requires angular position sensors possess strong reliability, low cost, compact, less installation, etc. Recently, numerous angular position sensors have been proposed in the literatures such as simple resistive potentiometer, capacitive effect systems, optical sensors and magnetic sensors. However, the resistive potentiometers always lead to abrasion and instability owing to the physical coupling between sensor and object. The volume of the capacitive sensors is too large to be integrated into compact structures. Optical sensors can realize high precision but are strict to the working environment which should not include dirt, dust, oil and so on. Considering the imperfections of those sensors mentioned above, magnetic sensors are widely applied in measuring angular position, which are mainly based on magnetostrictive, magnetoresistive and Hall effects attribute to advantages such as robust to complex working environment and no contact. Researchers engaged in improving structure and precision characterizations of magnetic sensors. However, they mainly focused on the types of relative angular position sensors rather than absolute ones. Furthermore, the position relationship between the magnetic drum and the magnetic point has a tremendous impact on the signals of sensor, which is unbeneficial to the installation of sensor.

Therefore, to bridge these gaps, a novel non-contact angular absolute position micro-sensor based on law of electromagnetic induction is presented. The linearity of the sensing system is improved by particularly designing spatial magnetic field distribution of asymmetric magnet, and Hall output signal is improved and converted by the second-order low-pass filter circuit into linear analog voltage, in order to achieve accurate measurement of the joint angle of the robot. Additionally, a very simple experimental set up based on Hall elements for absolute rotary position sensing system consisting of rotating axis, magnet ring, scale dial, signal processing circuit and data acquisition system is given. In the near future, it is essential not only to achieve the integration of sensors, but high degree matching of robot joints and sensing system. Most importantly, non-contact structure of this micro-sensor system has extensive application value and prospects for miniaturization and integrated design of robot joints.

\section{PRINCIPLE OF OPERATION}

The idea for implementation of absolute rotary sensor came from typical Hall effects. When current flows through the semiconductor, if applying a magnetic field in a direction perpendicular to the current, the both sides between semiconductors will exist voltage difference. When Lorentz force $f_{L}$ equals to electric field force $f_{E}$, electronic accumulation will reach a dynamic balance. Afterwards the electric field established between the two sides of semiconductor is called the Hall electric field $E_{H}$, and the corresponding voltage difference is called the Hall potential $V_{H}$. The relationship between $E_{H}$ and $V_{H}$ is as follows: 


$$
V_{H}=\frac{R_{H} I B}{d}
$$

Where $R_{H}$ is Hall constant, $\mathrm{d}$ is distance between two sides of semiconductor.

According to above expression, when the current $I$ is constant, Hall potential $V_{H}$ is proportional to the magnetic induction $B$. If there exists a certain angular between the direction of the magnetic field and the Hall element plane normal vector, actually effective magnetic field applying on the Hall element is the component of its normal direction $B \cdot \cos \theta$. (See Fig. 1) Then output of Hall voltage is given with:

$$
V_{H}=K_{H} I B \cdot \cos \theta
$$

In the process of rotating the magnetic field relative to the Hall chip, $\theta$ changes correspondingly and Hall potential will also change in respect to the normal magnetic field intensities. Although Hall potential which is analog signal will be easily disturbed by changes in the environment, through design of filter circuit and integrated circuit system on chip, signal quality could be improved. The non-contact characteristics of magnet and signal processing circuit could reduce the installation accuracy requirements and improve fault tolerance. And high-accurate measurement of robot joint absolute angular could be realized through algorithm calibration.

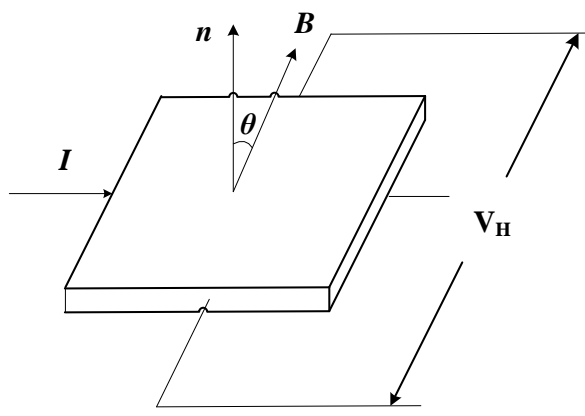

Fig. 1. The relationship between Hall output and the magnetic field angle

\section{THEORETIC DESIGN AND IMPLEMENTATION}

Absolute angular position micro-sensor uses split design method, with magnet and Hall elements mounting on two separate structural surfaces that are relatively rotating. The Micro - sensing system designed for robot joint angular measurement is shown in Fig.2. Thickness of magnetic ring and Hall chip is no more than $2 \mathrm{~mm}$. No matter what kind of electromechanical system, such as large robot systems or small robot dexterous hand joints, could easily installed the absolute micro-sensor system appropriately because of its advantage of small and light characteristics. Especially, this conceptual design makes great contributions to integration of sensing system and mechanical structure.
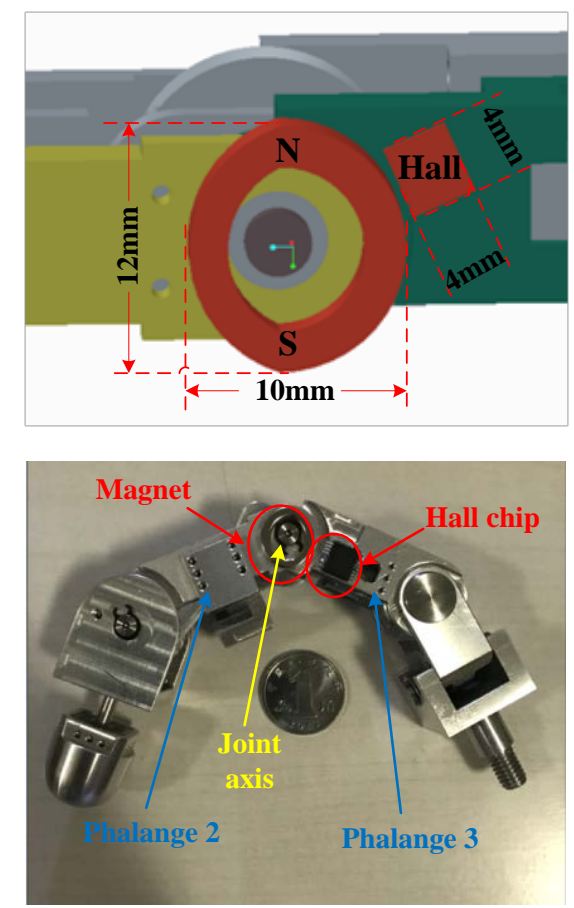

Fig. 2. Absolute position sensor designed for robot joint angular measurement

\section{A. Magnetic field design and analysis}

In order to improve Hall element sensitivity, it is essential to design magnetic field environment where magnetic flux density monotonously changes during angular measurement. In this section, structure of special magnet ring is designed and static magnetic field analysis is presented, verifying that magnetic flux density changes regularly within the limit of the rotation of robot dexterous hand joint.

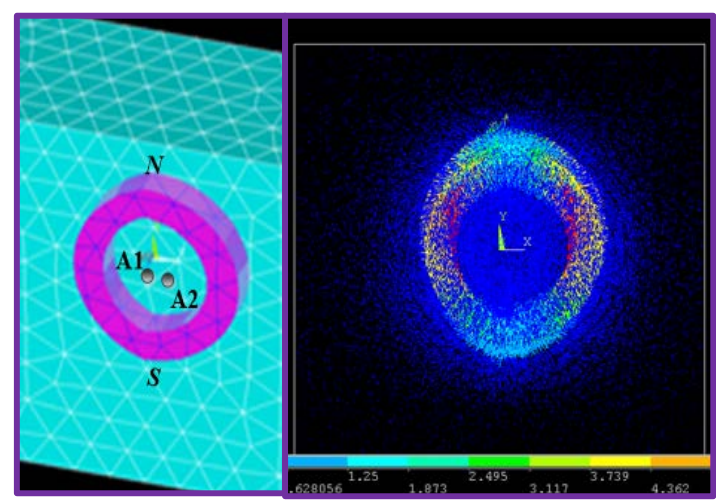

Fig. 3. finite element model and spatial magnetic induction vector distribution

Magnet ring is characterized by two portions. Left portion is a segment of a circle having a center point on axis A2. Right portion is a segment of a circle having a center point on axis A1. The north pole $\mathrm{N}$ of the Magnet is disposed at one intersection of the portions, and the south pole $\mathrm{S}$ of the magnet is disposed at the other intersection of the portions, face one another. The material of magnetic ring is neodymium - iron - 
boron alloy. The finite element model of the magnetic ring is created in ANSYS environment. Then the spatial structure unit, the relative permeability of the material and the coercively are defined. Afterwards, the spatial magnetic induction vector is obtained (See Fig.3)

The model of magnetic ring is defined as permanent magnet, and static magnetic field analysis is carried out. When robot joint axis is assumed to be in the $\mathrm{Z}$ direction, the spatial magnetic field intensity distribution is obtained in Fig. 4 and Fig.5.

As it can be observed in Fig.4, during rotating process of robot joint from 0 to 90 degrees, spatial magnetic field intensity along $\mathrm{X}$ component has a trend of monotonic attenuation. In Fig.6, the spatial total magnetic flux density distribution also increases regularly within the range of 360 degrees. Therefore, in actual joint angular measurement process, Hall element will be installed near the magnet ring. As magnet rotates with respect to Hall element about axis $\mathrm{A} 1$, the sensor maintains a constant distance from right portion of the magnet. The shape of the magnet and the placement of the Hall element provide a linear relationship between angular position changes and the change in magnetic field that is read by the sensor. Therefore, linear Hall voltage signal will be obtained with respect to angular changes within 90 degrees, and the absolute position of robot joint will be detected.

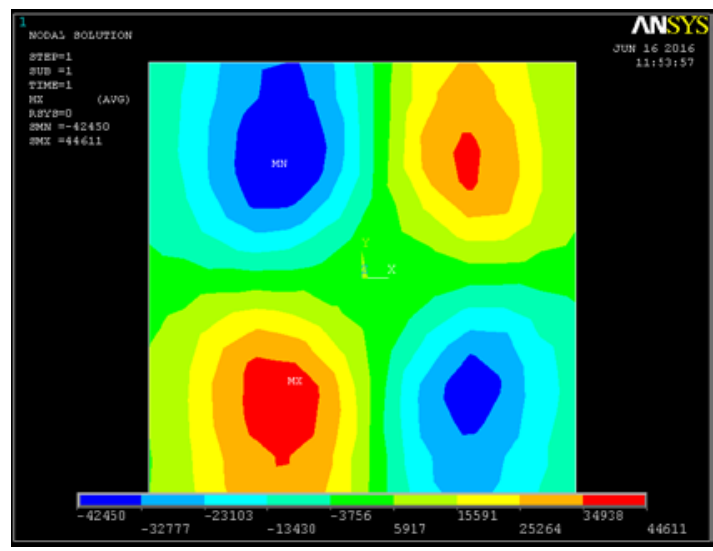

Fig. 4. Spatial magnetic field intensity along X component

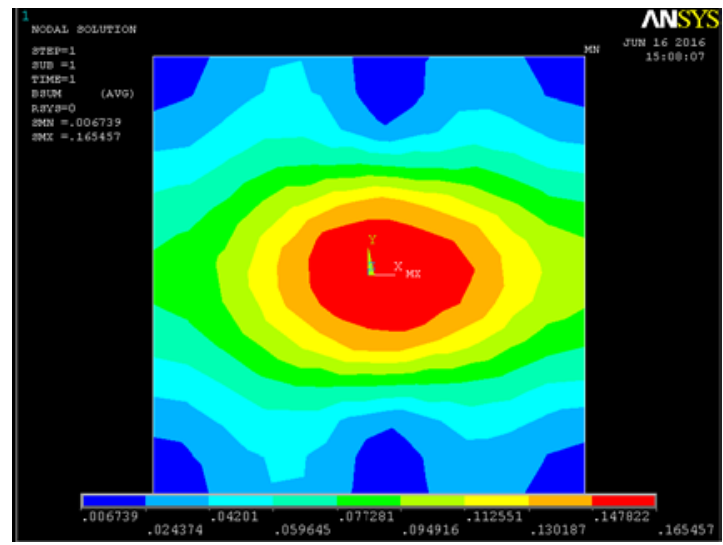

Fig. 5. Spatial total magnetic flux density distribution

\section{B. Signal processing circuit implementation}

As it can be observed in Fig.5, the range of spatial magnetic flux density distribution is from 243.74 to 949.16 Gaussian. In order to take full advantage of working magnetic flux density interval of Hall element, integrated Hall chip AS5045 is applied. The chip integrated Hall elements, power amplifying circuit and signal processing module. Based on AS5045, second order passive RC low-pass filter circuit is presented, converting the PWM signal into linear analog output signal (See Fig.6).

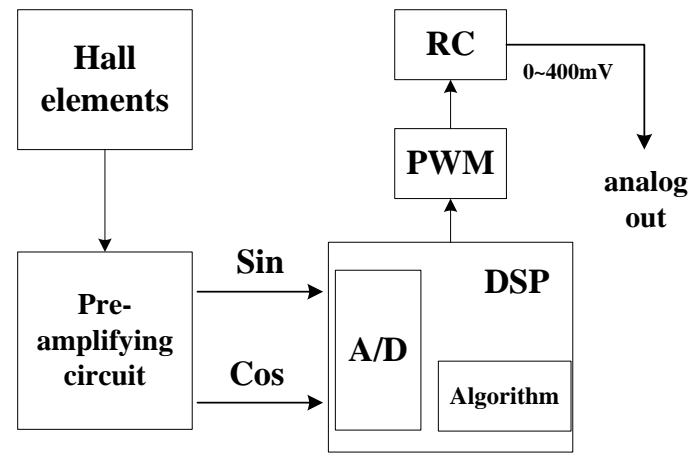

Fig. 6. principles of Hall signal processing and conversion circuit schematic

\section{ANALYSis Of SENSING System PERFoRMANCE}

\section{A. Experimental setup}

In the field of advanced robotics and automation, key performance indicators for evaluation of rotary position sensing system are sensitivity and linearity. The feasibility of the scheme is verified by the theoretical analysis of the overall structure of rotary position sensing system, including magnetic field design and analysis, and signal processing circuit implementation. In order to prove the effectiveness of the sensing system, the experimental setup for absolute rotary position measurement is presented based on previous principles of design (See Fig.7).

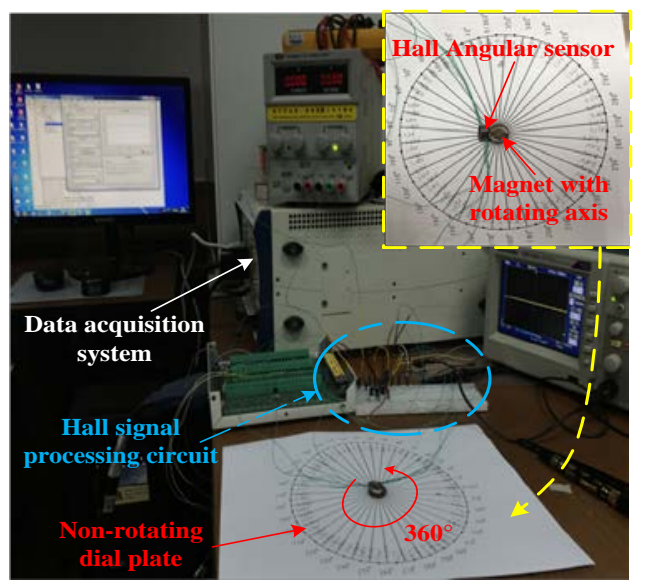

Fig. 7. Experimental setup for absolute rotary position measurement

The asymmetric magnetic ring is fixed to the end face of the rotary shaft, which could be able to rotate within 360 degrees. At the same time, the rotary axis passes through the 
center of a standard scale dial with a resolution of 5 degrees. Hall chip placed near the center of the magnetic circle and slightly lower than the height of the magnetic ring, could make full use of monotonic changes of magnetic field and obtain linear Hall voltage signal. Hall chip is connected to external signal processing and conversion circuit with $+5 \mathrm{~V}$ power supply. Data acquisition system is established in the host computer and analog output signal of sensing system is collected through the real-time controller at high frequencies.

\section{B. Results and discussion}

The Hall sensing system was tested through experimental setup with input of differential signal, in order to reduce common mode noise. During the process of rotary position measurement, the magnet ring is rotated at step of 5 degrees. The performance curve of sensing system output is obtained as in Fig.8 and Fig.9.

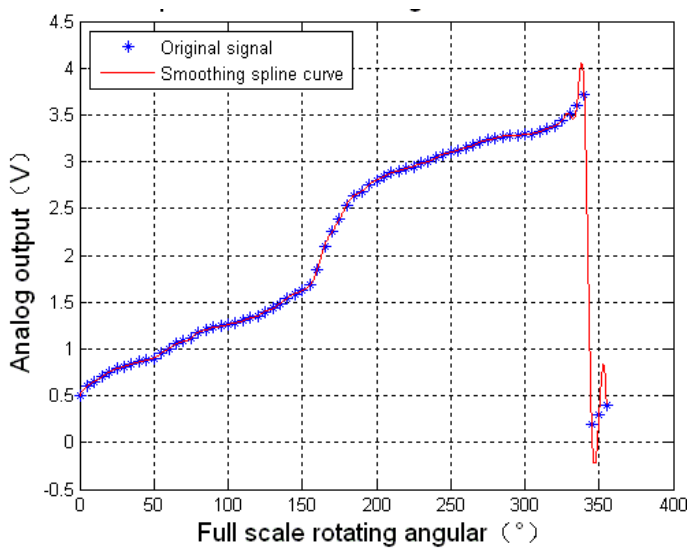

Fig. 8. output performance within 360 degrees

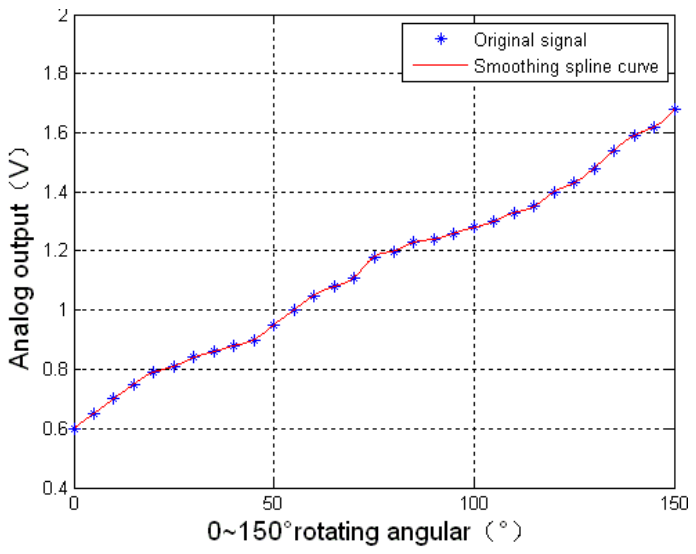

Fig. 9. output performance from 0 to 150 degrees

As it can be observed in Fig.8, due to spatial magnetic field distribution, the sensing system has a good linearity in the range of $0 \sim 150$ degrees and $185 \sim 330$ degrees, respectively. However, within scale of 160 180 degrees and 330 360 degrees, there is a steep change in the direction of magnetic induction lines or boundary magnetic field intensity changes unevenly, leading to Hall potential irregular jump. In Fig.9, data with good linearity is extracted and smooth interpolation is implemented. After performance testing, it can be easily found that rotary position sensor has high sensitivity and SignalNoise ratio. Output signal delay is no more than $96 \mu S$.

After the sensor fabrication is completed, calibration must be done in order to measure and adjust the response of a device. As sampling points still have a certain degree of non-linearity, 6th order Gaussian curve fitting is performed on discrete data points (See Fig.10). Afterwards, the linear analysis between measurement angular value and true position is presented, as it can be observed in Fig.11. As one can notice excellent linearity is achieved with R2 of 0.9992. Also, the key parameter is the slope of the characteristics which is very close to the ideal and is 0.9985 .

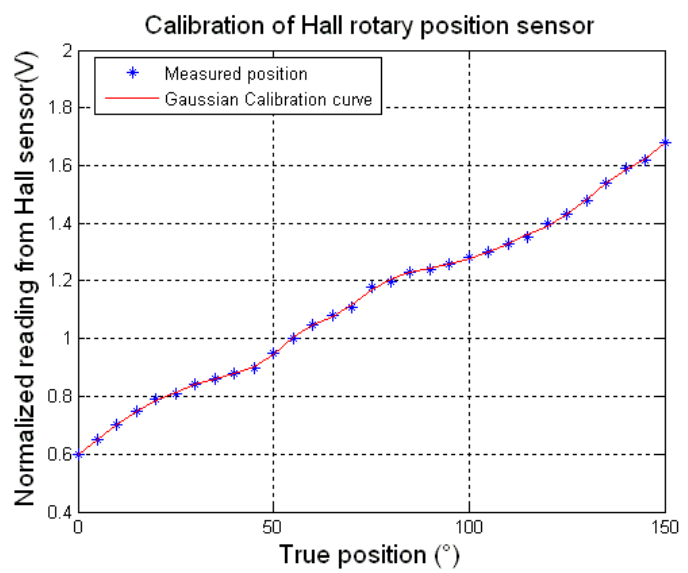

Fig. 10. Gaussian calibration of output signal

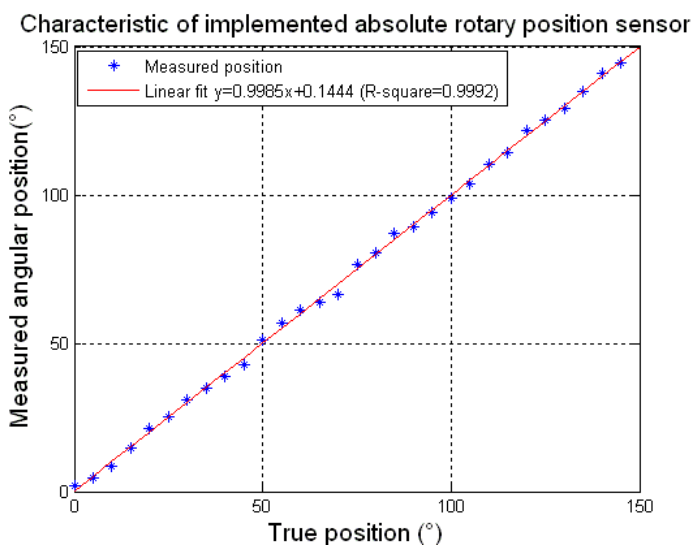

Fig. 11. Characteristic of implemented rotary position sensor

For expressing the precision of the device, differences in the angular position readings for several repeated measurements is presented (See Fig.12). As it can be noticed, a good performance is achieved with maximum characteristic drift around \pm 2.5 degrees. 


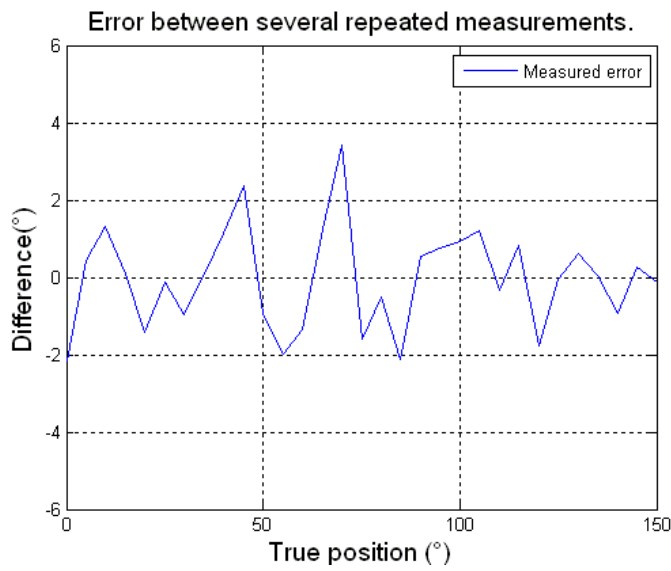

Fig. 12. Error between several repeated measurements

Operating speed of the sensor (in terms of rpm) was not tested. The main purpose of this paper is to demonstrate the idea and give a proof of concept of absolute position microsensor. Most importantly, it aims at solving difficulties that integration of robot joint and position sensing system in narrow space. However, for improving precision of sensor measurement, there are two ways to solve the problem. On the one hand, advanced algorithm could be developed to obtain high-quality signals. On the other hand, synchronous serial communication could be developed to convert analog signal into digital signal and avoid noise interference.

\section{CONCLUSIONS AND FUTURE WORK}

A novel absolute angular position micro-sensor based on the law of electromagnetic induction has been proposed in the paper. This sensor is easy to be installed and with highly integrated property which determine great application in industrial field. Excellent linearity has been accomplished with $\mathrm{R}^{2}$ of 0.9927 on the entire measurement range from 0 to 360 degrees. Accuracy of the proposed solution is below $\pm 5^{\circ}$. In this work, a simple experimental setup in order to demonstrate a proof of concept is given. It is assumed that even better performance can be accomplished with more precise equipment constructed or more effective signal processing methods. This will be one of the directions of the further research as well as actual testing of the sensor performance at different speeds of operation. However, much work should be done in the near future to improve performance of the sensor.
With rapid development of modern industrial automation, robotic integrated design requires rotary position sensors to have high precision, smaller size and longer life span. Method of non-contact sensing system is conducive to realize high degree matching of robot joints and sensing system. And noncontact structure of this micro-sensor system has extensive application value and prospects for miniaturization and integrated design of robot joints.

\section{ACKNOWLEDGMENT}

We thank Liu Shan and Zeng Guangshang for many insightful discussions. This work has been graciously funded under The 18th Institute China Academy of Launch Vehicle Technology.

\section{REFERENCES}

[1] Legrand, Bertrand; Dordet, Yves; Voyant, Jean-Yves; Yonnet, Jean-Paul. Contactless position sensor using magnetic saturation. Sensors and Actuators A: Physical, 106 (2003),pp.149-154.

[2] R. Pallàs-Areny, J.G.Webster, Sensors and Signal Conditioning, Wiley, New York, 2001, pp. 277-327.

[3] H. Son, K.-M. Lee, Two-DOF magnetic orientation sensor using distributed multipole models for spherical wheel motor, Mechatronics 21 (2011),pp.156-165.

[4] F. Vittorio, G. Alessio, M. Daniele, T. Andrea, Capacitive angularposition sensor with electrically floating conductive rotor and measurement redundancy, IEEE Transactions on Instrumentation and Measurement 55 (2006),pp.607-614.

[5] M. Gasulla, X. Li, Gerard C.M. Meijer, Jo W. Spronck, A contactless capacitive angular-position sensor, IEEE Sensors Journal 3 (2003),pp.607-614.

[6] J.-P. Yonnet, A. Foggia, S. Adenot, A differential magnetic position sensor, Sensors and Actuators A: Physical 81 (2000),pp.340-342.

[7] G. Malinowski, M. Hehn, F. Montaigne, A. Schuhl, C. Duret, R. Nautua, G. Chaumontet, Angular magnetic field sensor for automotive applications based on magnetic tunnel junctions using a current loop layout configuration, Sensors and Actuators A: Physical 144 (2008),pp.263-266.

[8] D. Wang, J. Brown, T. Hazelton, J. Daughton, 360 angle sensor using spin valve materials with SAF structure, IEEE Transactions on Magnetics 41 (2005),pp.3700-3702.

[9] Chris A. Ihrke. Hartland. MI(US); Lyndon Bridgwater,Houston, TX (US); Robert Platt, Houston, TX (US); Charles W. Wampler, II, Birmingham, MI(US); S. Michael Goza, League City, TX(US). ROBOTIC THUMB ASSEMBLY. United States Patent Application Publication, US 20110067520A1. Mar. 24, 2011. 